Neil Quinn Glasgow School of Social Work/Positive Mental Attitudes Project

Lee Knifton

Greater Glasgow NHS Board

\section{Promoting Recovery and}

\section{Addressing Stigma:}

\section{Mental Health Awareness}

\section{through Community \\ Development in a Low-} Income Area

encompasses a wide range of conditions, most commonly anxiety and depression, which account for the bulk of the morbidity at population level (WHO, 2001). Less common are more severe and enduring mental health problems such as schizophrenia and bi-polar illness, which have a significant impact at individual level and are more commonly associated with negative attitudes towards mental health.

Stigma towards people with mental illness increases the public health burden in a range of inter-related ways. Stigmatising and discriminatory behaviour in the community, in settings such as employment, education, housing and relationships, leads to social exclusion and hinders recovery. Stigma affects individuals' self-esteem and confidence about playing a part in society and in their communities, which increases isolation and reinforces the impact of exclusion. Stigma also affects the willingness of individuals to seek help at an early stage.

There have been a range of initiatives to address public attitudes towards mental health that have focused on advertising and media-based approaches to attitudinal change. In Scotland, a National Programme for Improving Mental Health and Well-Being has led this work through the See Me campaign (Scottish Executive, 2003). However, there has been less investment in co-ordinated, local anti-stigma programmes.

The Positive Mental Attitudes Project provides a model for developing a comprehensive approach to tackling stigma locally by delivering a broad range of anti-stigma work. With its focus on deprivation, it has the potential to be replicated in other low-income communities.

This paper describes the key elements of the model that are important for effective development and delivery. In doing so, it explores process issues in relation to the evolu- 
tion of the project, and considers the evaluation of its impact in relation to attitudes and behaviour among its target groups and on the service users involved in delivering the project.

The paper will explore emerging issues that have implications for the development of anti-stigma work in other low-income communities.

\section{Addressing stigma: the evidence}

It is recognised that negative labels applied to social groups can lead to widely held stigmatising attitudes and beliefs towards individuals associated with the group label. Stigmatising beliefs may exist in the wider population and among labelled individuals through negative self-image. This labelling, based on stereotypes, has been considered by several authors in relation to minority groups (Goffman, 1963), and more directly in relation to people with mental illness (Scheff, 1966). Link and colleagues (1989) developed a 'modified label theory', which emphasises the consequences of having a psychiatric 'label' and describes the impact of stigma on mental illness and recovery.

Understanding attitudes and stigma towards mental illness is complex. It is complicated by a range of factors including limitations on our self-awareness in relation to our attitudes, and the problems of 'expressed' attitudes in attitudinal studies. This means that we must treat with caution findings of research such as the Scottish Public Attitudes Survey (Scottish Executive, 2002), which found broadly positive expressed attitudes. Other research approaches which focus on the experience of people with mental ill-health have found that stigma and discrimination are much more prevalent (Berzins et al, 2003).

It is clear that there are persistent beliefs associated with labels of mental illness. In the Scottish Public Attitudes Survey (Scottish Executive, 2002), a significant minority of respondents reported beliefs that people with mental health problems are dangerous and do not recover, although some improvements in expressed attitudes are found in the second Scottish Public Attitudes Survey in 2004 (Scottish Executive, 2004). To some extent these beliefs are differentially associated with certain mental health problems, but they apply across problems with a common label of mental illness (Penn \& Martin, 1998).

Attitudes to mental health are affected by a number of factors interacting with one another, particularly positive personal contact with people with mental ill-health and other known factors, such as peer influences. In particular, the media play a key role in relation to attitudes to mental illness. Approaches to attitudinal change in relation to mental illness to some extent reflect these known influences; for example, the See Me campaign focuses primarily on media and advertising.

Measuring stigma is difficult, and correspondingly, measuring the impact of anti-stigma work and the relative contribution of these approaches is also challenging. An analysis of a broad range of evaluated approaches by Gale et al (2004) provides a helpful framework of what works most effectively in tackling stigma.

\section{Mental health service users' involvement in design, delivery and implementation of programmes}

There is a clear argument that mental health service users should lead the work, challenging stigma and dispelling stereotypes through their personal knowledge and experience. The evidence suggests that the best campaigners against discrimination are those who can challenge it personally and directly. Link et al (1989) found that people with mental health problems working collectively to address stigma were more likely to be effective. In addition, a study by Penn and Martin (1998) demonstrates that the most effective methods for reducing stigma involve promoting contact between the community and people with mental illness. The involvement of service users in the delivery of anti-stigma training programmes is one way of promoting positive contact.

\section{Multi-faceted approaches are the most effective way of reducing stigma and discrimination}

There is evidence to suggest that using a range of approaches is the best way of providing information and changing attitudes (Link \& Phelan, 2001). Using a range of intervention methods at a variety of times in a range of appropriate settings was shown to be effective in New Zealand and across the World Psychiatric Association global network of anti-stigma projects (Gale et al, 2004).

\section{Programmes should address behaviour change}

It is well documented that raising awareness and changing attitudes do not necessarily lead to a reduction in discriminatory behaviour. Programmes that aim to reduce stigma and discrimination must address discriminatory actions by individuals, groups and organisations. Effective approaches include addressing social inclusion and empowerment, providing support and skills to individuals, developing an environment of intolerance to prejudice, and ensuring that change is sustainable and supported by legislation. For example, the 
National Institute for Mental Health in England is working with the Disability Rights Commission to raise awareness of the rights of people with mental health problems under the Disability Discrimination Act (Social Exclusion Unit, 2004).

\section{Programmes should focus on the positive and promote recovery}

There is evidence that programmes that focus only on the negative aspects of stigma are less effective. There is more chance of success when programmes focus on the positive, by enhancing skills and building an individual's capacity to contribute, for example through community events which aim to reduce stigma in a fun way (Gale, 2004). Face-toface contact and community engagement are the most effective ways of reducing stigma and discrimination (Alexander \& Link, 2003).

\section{Clear, consistent messages need to be delivered, targeted to specific audiences}

Having clear and consistent messages is fundamental to having the desired impact (Gale et al, 2004). It involves focusing on a limited number of target audiences or projects, adopting social marketing principles to segment the audience and addressing anti-discrimination activities explicitly, and not as part of a general mental well-being programme.

\section{Long-term funding strategies and robust evaluation}

Changing the behaviour of the population is a long-term goal that will not be achieved overnight. Programmes that contribute most effectively to the evidence base are those which are planned, delivered and monitored over a number of years. It was found that the two key lessons from the Mind Out campaign in England were the need for longerterm funding strategies and robust evaluation (Social Exclusion Unit, 2004). As evidence is limited in this area, it is important for future programmes to add to the knowledge base by providing evidence of best practice.

We can now apply these principles in looking at a specific anti-stigma project, and examine the ways in which these ideas have been put into practice.

\section{Positive Mental Attitudes as a model of anti- stigma work}

Positive Mental Attitudes (PMA) was developed in 2001 by Greater Easterhouse Community Mental Health
Forum in partnership with a number of key partners from the Easterhouse area, including public sector agencies, voluntary sector organisations, community groups, Scottish Business in the Community and service users from the local area. The primary aim was to challenge the stigma surrounding mental ill-health. Greater Easterhouse is an area of multiple deprivations, as shown by a number of key indicators highlighted in a recent Community Health and Well-being Profile (NHS Health Scotland, 2004) of the local area. For example, 52\% of the 16-64 population are economically inactive, $37 \%$ of adults of working age are Income Support claimants, $58 \%$ of the adult population have no qualifications and $30 \%$ of adults state that they have a long-term limiting illness. These indicators are significantly above the Glasgow city and Scottish averages, and highlight the scale of deprivation in the locality.

The project is an integral part of the regeneration strategy of the local area, and operates as part of the Social Inclusion Partnership, the lead regeneration agency in the area. Given the poor health indicators in the area, improving health and well-being is seen as a key aspect of regeneration in Greater Easterhouse. The promotion of social inclusion for people with mental health problems through tackling stigma is a key priority of the health strategy group.

The programme was informed by an early baseline study of attitudes among the local community, and perceptions of service users. This baseline study suggested lack of awareness of mental health, prevalence of negative attitudes and a desire for more information and training.

As a result of this research, a programme of targeted anti-stigma initiatives was developed including:

- development of curriculum lessons in local secondary schools

- provision of information seminars and policy development for local employers

- anti-stigma training workshops for public service workers

- a range of 'social marketing' and arts work in the wider community.

Underpinning each strand of activity are the key principles of user participation and partnership working.

User participation: There is a high degree of service user involvement in the design, planning and delivery of each part of the programme. Sixteen service users from the local area are involved in the design 
and delivery of PMA. This contribution was recognised by employing service users as project workers, as part of the aim of promoting recovery.

- Partnership working: The involvement of a broad range of partners in planning and delivery, including youth workers, mental health professionals, schools, the local economic development company, social work staff, community planning organisations, the local arts development team and other generic regeneration agencies.

\section{Evaluation methodology}

From the outset, a robust monitoring and evaluation framework was built into the work of the project, which has collated written feedback from participants on the various initiatives within the programme. An external evaluation was commissioned at two years, with the aim of determining the effectiveness of the programme's development process and the impact of its interventions in addressing stigma in target groups in the short term, and encouraging and sustaining behaviour and organisation change in the longer term. The evaluation involved representation from all the key groups involved in the project.

A range of methods were used to evaluate the effectiveness of the programme, including:

- focus groups

- face-to-face interviews

- telephone interviews

- school post-course evaluation and analysis of project monitoring data.

Seven focus groups were led by an independent researcher using a discussion guide comprising:

- three groups with a total of thirty school children

- two groups with a total of sixteen service users, eight of whom had been involved in the project and eight who had not

- one focus group with six trainers and one focus group with community representatives.

A total of 56 structured telephone interviews were undertaken, which included 36 interviews with local employees who had received anti-stigma training and 20 interviews with local company representatives, 10 of whom had been involved in the workplace programme and 10 who had not. Twelve semi-structured face-to-face interviews were carried out with twelve key partners from a range of agencies, including primary care, local authority, local economic development company and voluntary sector organisations. Analysis of project monitoring data drew on 137 postworkshop evaluation forms and attendee demographic data.

\section{Programme output}

Over the following three-year period, the following activity was completed.

- A total of 150 lessons were delivered in three schools to more than 2,000 secondary school pupils aged 13-16 years, each pupil receiving a programme of two lessons. This curriculum programme was developed in partnership with Health Board and Education Department staff, and delivered by project workers, service users, youth workers and mental health and social work staff. The lessons focused on awareness of mental health issues for young people and attitudes towards mental ill-health. The decision was taken to mix mental ill-health with aspects of positive mental well-being.

- An ongoing series of 40 one-day training workshops was delivered to 500 workers from a diverse range of agencies, including social work, housing, primary health care, the police, education and benefits agencies, all of whom were employed in Easterhouse and worked directly with local people to provide key services. A training team was drawn from project workers, service users, community workers and mental health staff, who delivered the workshops after participating in a training for trainers programme. The workshops focused on exploring participant attitudes to mental health and identifying ways of combating stigma and discrimination. One feature was to support participants in exploring the stigma surrounding mental ill-health in relation to how stigma is experienced generally by other community groups.

- A programme of seminars and policy support was undertaken with 16 local employers drawn from the private, statutory and voluntary sectors. The seminars worked with managers and HR staff and focused on legal responsibilities to employees in relation to mental health, managing workplace stress, positive recruitment, policy development and accessing support for employees experiencing mental ill-health. The underlying aim was to develop a more supportive workplace and ethos to improve attitudes among the affected employees. 
- A range of social marketing and arts programmes were delivered, including website development and promotion, production of a local mental health resource directory, a poster campaign, video production, art and creative writing exhibitions, dramatic productions and media articles.

- Each of these initiatives was supported and steered by planning groups drawn from the original project partners and enhanced by drawing on specialist expertise such as Scotland's Health at Work, the local economic development company, marketing specialists, schools, arts development team and community planning organisations.

- Following the establishment of the project, the National Programme for Mental Health and Wellbeing was launched through the Scottish Executive. This helped to provide a focus on stigma as a national priority, and provided a strategic context for the project. A real synergy has developed between the local and national groups through, for example, local project staff working with the See Me campaign as media volunteers at national level.

\section{Impact of the programme}

\section{Impact on service users}

A sample of eight service users involved in the project reported an increase in confidence and skills as a direct result of being involved in the project. The group emphasised that they had all been involved in the design and delivery of the anti-stigma initiatives. They saw the development of confidence and skills among the service user group as an important success of the project and as contributing to their own recovery.

The focus group also highlighted the importance for service users of working collectively to address stigma. There was some indication that by working together to challenge stigma in society, we could move the perception of stigma towards a 'social problem' (Link et al, 1989) rather than an individual problem for those involved.

The evaluation also identified a belief among the group that negative attitudes to people with mental health problems in target groups in the local community had been challenged successfully. This proved to be a motivating factor for the service user group involved in delivering the work, and led to increased recruitment of others. This approach links to an underlying principle of the project, which was paying service users for their work in delivering training and awareness-raising, alongside paid professionals.
There were particular challenges in building capacity among the local users of mental health services involved in PMA. The very low educational attainment levels in the area (NHS Health Scotland, 2004), combined with the barriers associated with the mental health label, meant that personal development work was really important to the success of future work.

\section{Impact on target group attitudes and behaviours}

There are good signs from our analyses that there has been positive impact on expressed attitudes as a result of the project in the short and longer term (up to one year), and that there has been some impact on reported behaviours. There are important differences among the target groups in terms of reported attitude and behaviour change, and they are summarised below.

School pupils, in both the focus groups and the postcourse evaluation, reported an improvement in expressed attitudes to mental health. The key themes in the postcourse evaluation in relation to attitudinal change were that mental ill-health can affect anyone (75 of 137-55\%), lowered perception of dangerousness (44-32\%), and that mental ill-health can be overcome and people recover (38-28\%). In the focus groups, pupils also said that they believed that their training had made them more positive about mental health generally, and they would treat people more positively as a result.

Similarly, those who participated in the public sector anti-stigma training courses reported perceived attitudinal and behaviour change. The 36 telephone interviewees selfreported improvements in attitudes in relation to the following key themes:

- the importance of not labelling people (13 of $36-$ $37 \%)$

- the negative impact of stigma on individuals (8 $23 \%)$

- mental illness can affect anyone $(6-17 \%)$.

Importantly, more than two-thirds of respondents (69\%) felt that they had been able to put into practice changes to their behaviour attributable to their training. Behaviour changes reported by these 25 participants included taking more time with people with mental health problems (29\%), being less likely to label people negatively (21\%) and being more confident in supporting people with mental health problems (13\%).

Finally, although a smaller initiative, nine of the ten participating employers reported attitudinal changes and 
improved work practice as a result of engaging with the project's employer programme. Eight of the ten employers reported increased willingness to discuss mental health issues with their staff. Two of the ten have created a mental health policy, and another employer has created a display stand on mental health in the workplace.

These overall findings suggest a marked improvement in attitudes to mental health as a result of the training across all groups. We are aware that these results are limited, in that they are based solely on participants' subjective assessments of impact. Future analyses will explore other approaches to assessing effect.

\section{Impact of different approaches to tackling stigma}

The findings suggest that there is a core set of principles and tactics that proved effective in the methods of intervention adopted by the programme.

According to the various sources of data, the aspect of the training that appeared to work particularly well from the perspective of training participants was the involvement of service users and the sharing of their personal stories as part of the training. It appears that the power of the narrative of the person with mental illhealth, and their friends and families, to change attitudes is very significant. This bears out the more general concept that positive personal contact is important as a process within anti-stigma work.

The feedback from discussions with service users suggests that the project was successful in promoting positive images of mental health. This was particularly important, as service users were heavily involved in planning and delivery, and was a significant factor in reinforcing selfesteem. In particular, the personal stories of service users about recovery succeeded in engaging the training participants in thinking about their attitudes to mental health.

Evidence from the reviewed literature suggests that it is not as effective to deliver a programme that challenges stigma alongside general mental health promotion work. However, experience from the work of the project suggests that this is possible. For example, in the school work, the curricular lessons successfully included sessions on mental health promotion alongside challenging pupils' negative attitudes to people with mental health problems. Similarly, in the public sector training, feedback from participants resulted in the delivery of courses containing aspects of mental health awareness and promotion, in addition to challenging stigma. The usefulness of combining mental health promotion work with anti-stigma messages may be that participants are able to make a connection between their own mental health and their attitudes to people labelled as being mentally ill.

The baseline studies of attitudes and knowledge led the project to 'segment' the community into key priority groups. The groups were targeted with specific messages, and different approaches were adopted. The evaluation demonstrates overall effectiveness, although we are not yet in a position to compare the relative impact of adopting different approaches with each group. In addition, we have anecdotal evidence that using multiple approaches with one group, such as the workplace, was particularly effective, as suggested by Gale et al (2004). An example was a combined programme of training, policy development and arts initiatives delivered to employers. However, the monitoring framework has not yet been designed to measure this.

The focus groups with community representatives looked at the project's marketing strategy. The feedback was that awareness among the general public of the PMA anti-stigma initiative was low. This was supported by a separate study on the development of an anti-stigma training pack across the city, which found that among the nontargeted population in Greater Easterhouse the National Programme for Improving Mental Health and Well Being has a higher recognition profile. This is a predictable result; PMA is a relatively small project in terms of scope and capacity. A key lesson is the importance of the link between the National Programme and a local initiative like PMA. This supports the findings by Gale and colleagues (2004) which suggest that national programmes that support local activity demonstrate the most potent combination for efficacy. In the context of PMA, there are numerous examples of effective collaboration. For example, the local project distributed See Me publicity materials at local events such as the Greater Easterhouse Carnival, reinforcing national See Me media images. Another important link between local and national is the involvement of service users in delivering anti-stigma work within the project, enabling them to gain the skills to tackle stigma nationally through the media volunteer scheme.

The challenge of limited capacity was met by the engagement of a whole range of partners to deliver antistigma work. Feedback from the 12 partner interviews suggested that the partnership working was effective in delivery of the programme. Most of these partners did not have specific knowledge or experience of mental health, but they were able to offer expertise in their particular area for example working with young people or the business community. This was combined with the expertise offered by mental health service users, ensuring a team approach 
to addressing stigma drawing on a mix of different but complementary skills. Much of the capacity-building is still evolving; for example, youth workers, community workers, teachers and nurses have been trained and supported to deliver most of the school-based lessons and public sector training with ongoing support from the project. This has had the effect of massively increasing output, but the impact on achieving attitudinal and behavioural change needs to be measured. This is being investigated currently, and initial indications are quite positive. We are also monitoring the effects of delivering training for the trainers themselves, to see differences in the effects compared with service users and project staff.

This programme addresses problems of stigma in lowincome communities, where the prevalence of mental disorders is high. Stigma is also a concern is more affluent communities (Penn \& Martin, 1998), and the approaches to tackling stigma that we have outlined in this paper could be used. In doing so, it is important to recognise that there may be significant differences, such as absence of regeneration organisations to support the process, and differential initial skill levels among mental health service users involved in the delivery of the programme.

\section{Conclusions}

Stigma and discrimination limit the aspirations of people experiencing mental ill-health and can make it difficult for them to work, access services, participate in communities and enjoy family life. The literature demonstrates that there are a range of factors to take into account when developing anti-stigma work. Positive Mental Attitudes has been developed in one low-income locality and has evaluated well in terms of achieving attitudinal and behavioural change and promoting the involvement of a wide range of partners and service users in its development.

In particular, PMA has developed a range of training methods which have been effective with target groups. It was found that multiple and flexible approaches are needed for different groups, but that the personal narrative about recovery delivered by mental health service users is core to each approach adopted in the delivery of an anti-stigma programme.

The findings also suggest that it is possible, and perhaps desirable, to challenge stigma alongside the promotion of mental health and well-being. This differs from other approaches which focus solely on challenging stigma. Further research is needed to establish whether this approach dilutes or reinforces the anti-stigma message.

The evaluation also identifies a number of refinements to the approaches taken.

\section{Behaviour change}

A greater focus on behavioural change is needed. One of the key recommendations of the evaluation report was that a longer-term view is required on the project's funding. This was seen to be particularly important if the project is to be effective in supporting organisational change, which can take years to achieve (Axiom, 2004).

\section{Project marketing}

The evaluation suggests that the project needs to raise its profile in the local community, as the evidence suggests low awareness among those not actively targeted. The lack of more widespread awareness of the programme can be explained by the lack of specialist skills in social marketing methods, and the need to conduct more rigorous stakeholder analysis of the key target groups.

\section{Ongoing monitoring and evaluation}

External evaluation was positive about the project's evaluation framework. However, it was identified that there is a need to refine the evaluation methods. This would include establishment of participant knowledge and attitudes prior to the commencement of training, and a follow-up with individuals after their participation in training, to establish its effectiveness in changing behaviour. It would also be desirable to record information on individuals and organisations that choose not to participate in the programme, so that we can identify those with whom this approach does not allow us to engage effectively.

\section{Equality issues}

Equality in mental health has not been fully considered in relation to tailoring the training content to the needs of diverse groups in the community, such as asylum seekers. Differences in gender, ethnicity and age create particular mental health profiles, awareness and needs, which require significant additional consideration.

\section{Future developments}

The project hopes to expand across the East End of Glasgow, to integrate with the emerging Community Health Partnership in the East of Glasgow, and to develop its approaches across the city. A major challenge will be to retain the principle of local ownership of anti-stigma work while achieving the advantages of scale when rolling out a model of good practice. 
PMA offers an innovative model, which both promotes recovery and addresses stigma. The research suggests that the key lessons in replicating this model in other low-income communities are drawing on a broad coalition of community support and enabling service users to lead in the design and delivery of the anti-stigma programme.

\section{Contact details}

Neil Quinn, Glasgow School of Social Work, Jordanhill Campus, Sir Henry Wood Building, 76 Southbrae Drive, Glasgow, G13 1PP. Telephone: 0141950 3142. E-mail: neil.quinn@strath.ac.uk.

\section{Acknowledgements}

The authors acknowledge the following for their help: Linda McCall, Axiom Market Research and Consultancy for undertaking the project evaluation; Professor Andrew Kendrick, Glasgow School of Social Work, University of Strathclyde and Janice Scouller, Greater Glasgow NHS Board in the preparation of this paper. We would acknowledge the contribution of the service users and other key partners involved in the project evaluation.

\section{References}

Alexander, L.A. \& Link, B.G. (2003) The impact of contact on stigmatizing attitudes toward people with mental illness. Journal of Mental Health 12 (3) 271-89.

Axiom (2004) Positive Mental Attitudes Evaluation. St. Andrews: Axiom Market Research and Consultancy.

Berzins, K.M., Petch, A. \& Atkinson, J.M. (2003)

Prevalence and experience of harassment of people with mental health problems living in the community. British Journal of Psychiatry 183 526-33.

Gale, E. (2004) More than lip service: securing lasting change in the fight against stigma and discrimination. Journal of Mental Health Promotion 3 (3) 5-7.

Gale, E., Seymour, L., Creapaz-Keay, D., Gibbons, M., Farmer, P. \& Pinfold, V. (2004) Scoping Review on Mental Health and Anti-Stigma and-Discrimination: Current activities and what works. Leeds: NIMHE.

Goffman, E. (1963) Stigma: Notes on Management of Spoiled Identity. New Jersey: Prentice-Hall.

Link, B.G., Cullen, F.T., Struening, E. et al (1989) A modified labeling theory approach to mental disorders: an empirical assessment. American Sociological Review 54 400-23.

Link, B.G. \& Phelan, J.C. (2001) Conceptualising stigma. American Sociological Review 27 363-85.

NHS Health Scotland (2004) Eastern Glasgow: A community health and well-being profile. Edinburgh: NHS Health Scotland.

Penn, D.L. \& Martin, J. (1998) The stigma of severe mental illness: some potential solutions for a recalcitrant problem. Psychiatric Quarterly 69 (3) 235-47.

Scheff, T. (1966) Being Mentally Ill: A Sociological Theory. Chicago, Aldine.

Scottish Executive (2002) Well? What do You Think? A national Scottish survey of public attitudes to mental health, well-being and mental health problems. Edinburgh: Scottish Executive.

Scottish Executive (2003) National Programme for Improving Mental Health and Well-Being: Action Plan 2003-2006. Edinburgh: Scottish Executive.

Scottish Executive (2004) Well? What do you think?: The second national Scottish survey of public attitudes to mental health, well being and mental health problems. Edinburgh, Scottish Executive.

Social Exclusion Unit (2004) Social Exclusion and Mental Health. London: Office of the Deputy Prime Minister.

World Health Organisation (2001) The World Health Report 2001. Mental health: new understanding, new hope. Geneva: WHO. 